\title{
Identificación de estrategias de capacitación en pymes de la Ciudad de México
}

\author{
Identification of training strategies in SMEs \\ in Mexico City
}

Jorge Luis Alvarez Morales ${ }^{1}$ y Dania Ramírez Herrera ${ }^{2}$

Fecha de recepción: 31 de enero de 2021

Fecha de aceptación: 28 de abril de 2021

1 - Nacionalidad: Cubana. Grado: Licenciatura en Ingeniería Industrial. Estudiante de la Maestría en Ciencias en Estudios Interdisciplinarios para Pequeñas y Medianas Empresa. Adscripción: Instituto Politécnico Nacional (IPN) (1) ORCID: https://orcid.org/0000-0002-3733-6355 | Correo electrónico: jorgelalvarez90@gmail.com

2 - Nacionalidad: Mexicana. Grado: Maestría en Ciencias en Administración de Negocios. Adscripción: Instituto Politécnico Nacional (IPN). (1) ORCID: https://orcid.org/0000-0001-6686-6155 | Correo electrónico: dara1991@live.com.mx

https://creativecommons.org/licenses/by-nc-sa/4.0/ 


\section{Resumen}

Las pequeñas y medianas empresas son de gran importancia para la economía mexicana. Sin embargo, estas organizaciones presentan diversas problemáticas, como la falta de capacitación. Por ello, esta investigación tiene como objetivo identificar las estrategias de capacitación y los elementos que componen su desarrollo. La metodología que se sigue es de carácter cualitativo. La recolección de información se ejecutó mediante observaciones y entrevistas semiestructuradas a propietarios-gerentes y trabajadores de una pequeña y una mediana empresa de la Ciudad de México, ambas dedicadas a la fabricación, prestación de servicios y comercialización de equipos. Con base en la teoría fundamentada y con el apoyo del software ATLAS.ti se realizó la codificación y análisis de la información. Los resultados permitieron identificar tres tipos de estrategias de capacitación: hacia el ahorro de recursos, hacia los conocimientos y habilidades, y las combinadas. Además, se definieron tres elementos que influyen en dichas estrategias.

Palabras clave: estrategias de capacitación, proceso de capacitación, agentes, habilitadores, pymes.

\section{Abstract}

Small and medium-sized companies are of great importance to the Mexican economy. However, these organizations present several problems, such as a lack of training. Therefore, this research aims to identify training strategies and the elements that make up their development. The methodology had a qualitative nature. The information collection was carried out through observations and semi-structured interviews with owner-managers and workers of one small-sized and one medium-sized company in Mexico City, both dedicated to the manufacture, provision of services, and commercialization of equipment. Based on the Grounded theory and with the support of the ATLAS.ti software, the coding, and analysis of the information were developed. The results allowed the identification of three types of training strategies: towards saving resources, towards knowledge and skills, and combined. In ad-

dition, three elements that influence the development of these strategies were defined.

Keywords: training strategies, training process, agents, enablers, SMEs. 


\section{Introducción}

$\mathrm{L}$

as pequeñas y medianas empresas (pymes) son de suma importancia a nivel mundial, debido a su aporte en indicadores como ingresos generados y creación de empleos, acorde con la Oficina Internacional del Trabajo (OIT, 2015). Las pymes participan en el 45\% de la generación del Producto Interno Bruto, pero estas empresas son hasta $70 \%$ menos productivas que las grandes, según la Organización Mundial del Comercio (OMC, 2016). Es por ello que, enfocados al fomento de estas organizaciones, múltiples gobiernos realizan esfuerzos económicos para lograr el desarrollo de estas empresas (Ayyagari et al., 2014). El Banco Mundial reconoce a las pymes como parte fundamental de su estrategia orientada a promover el empleo, el desarrollo de las cadenas de valor, la inclusión social y económica (Independent Evaluation Group, 2019). Sin embargo, el crecimiento de las pymes está determinado por numerosos factores como: condiciones económicas locales, las habilidades administrativas de los propietarios, la cultura organizacional, el acceso a financiamientos o el conocimiento y la capacitación adecuada (Smit y Watkins, 2012).

Sobre la capacitación en estas organizaciones, Padachi y Bhiwajee (2016) plantean que mientras más grandes son las empresas, las mismas son más propensas a realizar formación laboral. Ello se debe a elementos como: que las grandes empresas tienen mayor facilidad de coordinación de sus procesos, actividades y un presupuesto superior para invertir (Almeida y Aterido, 2015).

Las pymes en México, sin incluir las micro empresas, generan el 30.6\% del personal ocupado y el 38\% de los ingresos nacionales, según el Instituto Nacional de Estadística y Geografía (INEGI, 2020). Sobre la capacitación como problemática que afrontan las pymes mexicanas se encuentra que declaran no realizarla el 44.9\% de estas entidades económicas (INEGI, 2018). Según este mismo informe, las causas más frecuentes por la que no realizan capacitación son: creer que el conocimiento y la habilidad del trabajador son suficientes, se contrata personal ya capacitado, costos elevados, pensar que esta interrumpe el trabajo y que se impartió en años previos. No obstante, la capacitación se recoge en la legislación vigente como obligatoria para los empleadores y empleados, dictado así en la Ley Federal del Trabajo en su artículo 153-A (Congreso General de los Estados Unidos Mexicanos, 2012).

Así mismo, en estudios internacionales se hallan también barreras relacionadas al proceso de capacitación en pymes. En este sentido, Sethi (2018) señala como principales obstáculos: la escasez de mano de obra que generan la participación en actividades de capacitación, la renuncia de los trabajadores entrenados como resultado de mejores ofertas laborales, la preferencia de los trabajadores por ganar dinero, que no hay garantía del retorno de la inversión (ROI), la ausencia de recursos, pérdida de tiempo, etc. Bai et al. (2016) sostienen que los gerentes están más preocupados por el retorno de ganancias a la empresa por parte de la capacitación que por los beneficios a los trabajadores, además de aspectos externos como la calidad y la ubicación de la formación. Por su parte, Padachi y Bhiwajee (2016) determinan inhibidores como: la percepción de los gerentes sobre la capacitación, las presiones externas, el ciclo de capacitación, el diseño, los costos y el financiamiento.

Por otra parte, aquellas pymes que sí realizan capacitación no siguen los procedimientos y lineamientos descritos en la literatura, lo que impide maximizar sus beneficios (Mendoza, 2005). La mayoría de estas organizaciones no realizan un proceso formal de capacitación con el diagnóstico de 
necesidades y la evaluación final del mismo (Pauli, 2019). Siendo, según Padachi y Bhiwajee (2016) la capacitación informal la forma más común de realizar el aprendizaje en estas empresas. Además, en el caso de las organizaciones mexicanas es frecuente que la capacitación no sea ejecutada con consistencia y seriedad (Siliceo, 2004).

Por lo tanto, las pymes al no llevar a cabo capacitación o no realizar esta de forma adecuada, tomando en cuenta los principios básicos descritos en la literatura, no pueden obtener los resultados que esta ofrece. Estudios en estas organizaciones resaltan los beneficios de la adecuada capacitación como son: aumento en la productividad (Ciriaci, 2016; Padachi y Bhiwajee, 2016; Pauli, 2019), la producción de innovación (Ciriaci, 2016, Susomrith et al., 2019), incremento en el desempeño (Padachi y Bhiwajee, 2016; Pauli, 2019), entre otros. Además, la formación trae beneficios a los trabajadores por la adquisición de competencias profesionales, el aumento en la satisfacción y la creación de condiciones para un mejor desempeño laboral (Jehanzeb y Bashir, 2013).

La necesidad de planificar y ejecutar procesos de capacitación formales en el contexto de las pequeñas y medianas empresas, no constituye la única problemática de esta temática. Así mismo, la literatura señala que la capacitación y el desarrollo de recursos humanos en pymes es un tema descuidado, en el cual no se ha realizado suficiente investigación (Nolan y Garavan 2016; Short y Gray, 2018). La revisión sistemática de literatura realizada por Nolan y Garavan (2016a), destaca que se utiliza un grado limitado de perspectivas teóricas y conceptuales, existiendo un gran potencial de investigaciones futuras acerca de aspectos como: la capacitación formal e informal, el enfoque estratégico de la misma y metodologías que aborden más los participantes y su contexto.

Teniendo en cuenta los elementos planteados anteriormente: la importancia de las pymes, el gran número de estas empresas que no capacitan en México, la falta de adopción de procedimientos adecuados que permitan obtener ventajas de la formación y la brecha de oportunidad en la literatura actual; se considera que es necesario realizar un estudio sobre el tema.

En este sentido, el objetivo de la presente investigación consiste en: identificar las estrategias de capacitación en las pymes estudiadas, así como la definición de los elementos que intervienen en el desarrollo de dichas estrategias.

Por lo tanto, para guiar esta investigación se busca dar respuesta a las siguientes preguntas:

¿Qué elementos son la base para el desarrollo de estrategias de capacitación en las pymes estudiadas? ¿Cuáles son las estrategias de capacitación que adoptan las pymes estudiadas?

El presente artículo está estructurado, primeramente, por un marco teórico sobre: la administración de la capacitación como parte de la actividad de recursos humanos, el proceso de capacitación y los aspectos sobre la formación en las pymes. En segunda instancia, se detalla el proceso de desarrollo de una metodología cualitativa con el uso de la teoría fundamentada y una muestra de dos pymes que realizan capacitación constante, ambas dedicadas a la fabricación, prestación de servicios y comercialización de equipos. Cabe destacar que con este trabajo se persigue la particularidad y no la generalización de los resultados, tal como señala Creswell (2014) sobre las investigaciones cualitativas. Aunque se entiende que la misma, por sus características, puede ser un punto de partida de futuros estudios. Por último, se exponen los resultados que dan respuesta a las interrogantes planteadas, así como la discusión con la literatura. 


\section{Marco teórico}

La teoría sobre el tema que se presenta en este punto está compuesta por: la capacitación dentro de la gestión de los recursos humanos, el proceso de administración de la capacitación y las particularidades de esta actividad en las pymes.

\subsection{La capacitación dentro de la actividad de recursos humanos}

El propósito de la administración de recursos humanos, donde queda implícita la capacitación, es el mejoramiento de las contribuciones de las personas a la organización (Werther y Davis, 2008), pues esta tiene la capacidad de alinear a los empleados de una empresa con la estrategia del negocio (Mondy, 2010).

En la literatura se describen varias definiciones de capacitación que comprenden los términos de: formación, entrenamiento y desarrollo (Mendoza, 2005; Werther y Davis, 2008). La capacitación o formación, sin embargo, puede definirse como capacitación y desarrollo, la primera centrada en los conocimientos, habilidades y aptitudes que requiere el trabajador para su puesto actual. Mientras que la segunda tiene el mismo propósito que la capacitación, pero orientada al futuro del trabajador en la empresa (Mondy, 2010; Noe, 2017). Por lo tanto, para alcanzar sus objetivos toda empresa requiere de su capital humano con una formación adecuada, con personal especializado en nuevas o futuras tareas, para lograr niveles de rendimiento y productividad que hagan de la organización un competidor en el mercado (Vásquez-Torres, 2018).

Si bien la capacitación provee múltiples beneficios, la forma de maximizar estos es mediante el desarrollo de un proceso de forma estructurada y cumpliendo principios básicos. Esta debe partir de la idea de que la capacitación laboral es efectiva solo si el aprendiz aplica esta para mejorar el rendimiento en el trabajo (Jain, 2014). La formación en una empresa es proporcionada dentro de un programa de capacitación, es por eso que en una primera instancia se lleva a cabo el análisis de las áreas problemáticas (Ostrowski et al., 2014). Si se identifican necesidades de capacitación, entonces se inicia un proceso de capacitación. El cual comienza con el diagnóstico de las necesidades de capacitación, luego el diseño e implementación y por último la evaluación (Aragón-Sánchez et al., 2003; Jabbour, 2013; Ostrowski et al., 2014).

\subsection{Proceso de administración de la capacitación}

La primera etapa del proceso de formación antes mencionado comprende el diagnóstico de necesidades de capacitación (DNC). El cual, según Jabbour (2013) busca examinar las insuficiencias de capacitación y determinar qué habilidades se requieren desarrollar. De acuerdo con Noe (2017), estas necesidades se deben analizar a tres niveles: organizacional, de las tareas y de las personas. Para determinar estas se utilizan métodos como: la evaluación del desempeño, las entrevistas, la observación, análisis de puestos, entre otros (Tao et al., 2006). Estas necesidades se clasifican de acuerdo a su fácil 
identificación como: encubiertas y manifiestas (Mendoza, 2005). Según Ostrowski et al. (2014), establecer las necesidades de capacitación adecuadamente, es la base esencial para determinar qué estrategia se seguirá en el próximo paso.

Luego del diagnóstico se encuentra la fase de diseño, la que involucra la toma de decisiones sobre la asignación de roles y responsabilidades (Jabbour, 2013). Los elementos que componen esta etapa son: los objetivos, los métodos de formación y principios de aprendizaje, las técnicas de formación y la posibilidad de practicar (Blume et al., 2010). Hodges (2002) sugiere que, como punto de partida, los objetivos se deben trazar a tres niveles: desde lo que se espera para el negocio, para el desempeño de los participantes y para el conocimiento que deben adquirir los trabajadores una vez concluida la capacitación. Además, Dessler y Varela (2011) señalan que las metas establecidas en esta etapa serán los puntos de partida de la evaluación de la capacitación.

Dentro de los métodos para impartir la capacitación que se recogen en la literatura los más comunes son: aprendizaje electrónico (e-learning), estudio de casos, rotación de puestos, juego de roles, simulaciones, observación de profesionales, entrenamiento de equipos, entre otros (Ostrowski et al., 2014; Mondy, 2010).

La etapa posterior al diseño es la implementación, la cual consiste en la puesta en práctica de lo que se planteó hacer (Aragón-Sánchez et al., 2003). Por otra parte, Gainey y Klass (2003) sugieren que las organizaciones pueden obtener beneficios si subcontratan el servicio de diseño e implementación de programas de capacitación a organizaciones especializadas en el tema. Pues, se debe tener en cuenta que la capacitación genera un cambio, lo que puede traer consigo resistencia por parte de los trabajadores (Mondy, 2010).

La última etapa del proceso es la evaluación de la capacitación, la cual busca identificar hasta donde se satisface el DNC (Ostrowski et al., 2014). Existen múltiples metodologías para la evaluación de la capacitación, las cuales están fundamentadas sobre la base de lo planteado por Kirkpatrick en los años sesenta (Jain, 2014), el cual propone cuatro niveles de evaluación que deben ser respetados por su orden: (1) reacción: busca conocer cómo reaccionó el participante al programa; (2) aprendizaje: se mide si el participante tuvo al menos: cambio de actitudes, mejora en el conocimiento y/o aumento de las habilidades; (3) comportamiento: el cambio de comportamiento del participante; y por último (4) resultados: el aporte que tuvo la capacitación en el desempeño de la empresa (Kirkpatrick y Kirkpatrick, 2006). Otras metodologías como las de Hodges (2002) y Noe (2017) añaden el factor del cálculo del ROI como elemento fundamental de la evaluación, el cual consideran los autores podría ser beneficioso para la toma de decisiones.

Todas las etapas antes mencionadas del proceso de capacitación deben ser sistemáticas, pero lo suficientemente flexibles para adaptarse a las necesidades que demande la empresa (Noe, 2017).

\subsection{A spectos de la capacitación en las pymes}

El comportamiento de las pymes en referencia a la capacitación, dista del que siguen las grandes empresas (Devins y Johnson, 2003; Short y Gray, 2018), pues estas organizaciones no cuentan en su gran mayoría con departamentos especializados de recursos humanos y no tienen un presupuesto destinado a la actividad de formación (Short y Gray, 2018). 
Por su parte, Barrett (2015) encuentra que los principales motivos que hacen que los propietarios-gerentes realicen DNC son: los requisitos legislativos o reglamentarios, la evaluación del empleado, el lanzamiento de nuevos productos o servicios, la creación de nuevos puestos de trabajo y para responder a las necesidades del mercado. No obstante, un estudio realizado por Pauli (2019) halla que las pymes que realizaron procesos de capacitación alineados a las exigencias de la literatura, obtuvieron mejores resultados y aumento en los indicadores de desempeño. Estos casos son poco frecuentes, pues de acuerdo a Nolan y Garavan (2016), el diseño e implementación de la capacitación en las pymes es típicamente realizado por los fundadores o el propietario-gerente, el cual no cuenta frecuentemente con los conocimientos sobre el tema.

Las pymes tienen un horizonte temporal corto debido a sus altos niveles de incertidumbre y por el contrario los beneficios de la capacitación tienden a observarse a largo plazo, lo que provoca en muchas ocasiones que la formación no sea atractiva para estas organizaciones (Kotey y Folker, 2007). En consecuencia, estas empresas tienden a confiar en métodos de formación que se derivan exclusivamente de experiencias obtenidas en el trabajo para el desarrollo de habilidades y conocimientos de sus empleados, por lo que suelen tener trabajadores menos comprometidos (Susomrith et al., 2019).

Referente a las estrategias que las pymes siguen para desarrollar acciones de capacitación, Bencsik y Sólyom (2011) identifican tres categorías. Primero, la estrategia basada en elementos convencionales donde predomina el aprendizaje externo. La segunda, de forma opuesta, donde existe un intercambio de conocimiento de forma interna predominantemente. La tercera y última, hace referencia a la combinación de ambas estrategias antes mencionadas.

\section{Metodología}

Las investigaciones sobre la capacitación y el desarrollo de las pymes han estado enmarcadas por enfoques cuantitativos y positivistas; descuidando elementos de investigaciones cualitativas como: la perspectiva de los participantes y el contexto de estas organizaciones (Nolan y Garavan, 2016b). Por lo tanto, para el desarrollo de esta investigación se sigue una metodología cualitativa, la cual permite estudiar el fenómeno desde la experiencia de los participantes (Creswell, 2014) e interpretar este en su contexto (Blaxter et al., 2002).

\subsection{Muestra}

La muestra seleccionada, acorde con la clasificación planteada por Crowe y Sheppard (2012) es no probabilística y dentro de esta se utiliza el método por conveniencia. Considerando el tipo de muestreo, se definieron un grupo de criterios para la búsqueda de empresas que se desempeñaran en la Ciudad de México. De esta forma se garantiza definir una población y a partir de esta seleccionar la muestra que logre una similitud entre los lugares de estudio, de tal manera que exista la menor variación posible (Eisenhardt, 1989). Los criterios fueron: 
1. Tamaño de la organización: Pequeñas o medianas empresas, según la estratificación que en México recoge la Secretaría de Economía (2009).

2. Años de vida: Organizaciones que contaran con alto grado de experiencia, mayores de 15 años de vida.

3. Actividad de capacitación: Empresas que declararan realizar actividades de formación continua a sus trabajadores.

4. Sector: Entidades económicas que se dedicaran a la actividad conjunta de comercialización, manufactura y prestación de servicios. Específicamente en la rama de equipamientos industriales.

5. Disponibilidad de los participantes: Organizaciones donde se permitiera realizar entrevistas a los propietarios-gerentes y trabajadores de todas las áreas. Pues, según Stake (1999), este es un elemento importante para la selección de un lugar donde investigar.

Con los cuatro primeros criterios se realizó una búsqueda en catálogos por Internet, encontrándose un total de 28 entidades. Las cuales, de acuerdo con sus páginas Web y otros sitios consultados, las mismas cumplían con los criterios de inclusión. A las pymes identificadas se les enviaron correos con peticiones formales para participar en el presente estudio. De estas empresas estuvieron de acuerdo en participar solamente tres. De ellas, dos permitieron realizar entrevistas a todas las personas que se consideraran conveniente por parte de los investigadores, cumpliendo con el criterio 5. La tercera empresa se excluyó del estudio, pues solo se pudo acceder a una entrevista al gerente de la misma, lo que no permitió garantizar la validez de la información a partir de la triangulación con otras fuentes de información.

En el Cuadro 1, se muestran las características de estas dos empresas. Para garantizar la confidencialidad como parte de las consideraciones éticas, los nombres de las pymes y de los participantes fueron sustituidos por códigos (Creswell, 2014).

\section{Cuadro 1}

Características de las empresas estudiadas

\begin{tabular}{|l|l|l|}
\hline CARACTERÍSTICAS & EMPRESA IM & EMPRESA EJ \\
\hline Tamaño & Mediana (52 trabajadores). & Pequeña (12 trabajadores). \\
\hline Años de vida & 63 años (fundada en 1957). & 34 años (fundada en 1986). \\
\hline Actividades que realizan & $\begin{array}{l}\text { Comercialización de equipos } \\
\text { hidráulicos (bombas, fuentes, sistemas } \\
\text { contra incendio, etc.), servicios de } \\
\text { mantenimiento, diseño y fabricación } \\
\text { de tableros de control para sistemas } \\
\text { hidráulicos. }\end{array}$ & $\begin{array}{l}\text { Fabricación, comercialización y prestación } \\
\text { de servicios a equipamientos eléctricos } \\
\text { (transformadores, supresores de pico, } \\
\text { UPS, etc.). }\end{array}$ \\
\hline
\end{tabular}

Fuente / Elaboración propia

\subsection{Recolección de la información}

Dentro de los métodos de recolección de datos en investigación cualitativa, planteados por Creswe11 (2014), se usaron la entrevista y la observación. Las entrevistas se realizaron de forma semiestructurada a trabajadores y propietarios-gerentes de ambas empresas. Sobre este método de recolección, en una primera instancia, se realizó una prueba piloto a tres trabajadores de distintas áreas de la empresa 
IM, con la finalidad de corregir preguntas que no fueran precisas. Acorde con Crowe y Sheppard (2012), se garantiza de esta forma la calidad en la recolección de los datos.

En la empresa IM se entrevistaron a 16 trabajadores y los 2 propietarios-gerentes. Mientras que en la empresa EJ se entrevistó al propietario-gerente y 6 trabajadores. En ambas empresas se realizaron entrevistas a empleados de todas las áreas, cada una tuvo una duración de 25-35 minutos aproximadamente. En el caso de las que se les realizaron a los propietarios-gerentes, demoraron entre 45-60 minutos aproximadamente. Esta forma de recolección de información se llevó a cabo de forma presencial y mediante llamadas telefónicas.

Las observaciones se realizaron en formaciones, tanto formales como informales. Las capacitaciones informales fueron a través de dos cursos online de varias sesiones ofrecidos por proveedores. En cambio, la capacitación informal fue presenciada en la empresa IM, en la formación recibida por un trabajador de nuevo ingreso. Se tomaron notas de campo como participante y como observador, como sugiere Creswell (2014).

\subsection{Análisis de la información}

En la medida que se realizó la recolección de información esta fue transcrita, codificada y analizada. Se compararon constantemente los códigos generados con los códigos existentes, con las categorías que se obtuvieron y con las notas que se tomaron durante el análisis; garantizando así la comparación constante (Corbin y Strauss, 1990).

Mediante la codificación de los datos se buscó reducir el volumen de información para poder analizar la relación entre esta (Birks et al., 2013), además que contribuyó al desarrollo creativo de manera sistemática (Corbin y Strauss, 2002). Para realizar la codificación se siguieron los tipos de codificación descritos en el diseño de teoría fundamentada: abierta, axial y selectiva (Corbin y Strauss, 1990, 2002):

1. Primero, se realizó el análisis de la información transcrita, la cual se efectuó entre párrafos $\mathrm{u}$ oraciones en la medida en que finalizó una idea con un mismo sentido. A partir de la separación de la información se encontraron categorías y subcategorías preliminares. El objetivo principal fue reducir el volumen de información a analizar a partir de una primera idea de categorización.

2. Luego, se relacionaron las diferentes subcategorías con categorías específicas. Para descubrir las relaciones se buscó dar respuesta a las interrogantes: por qué sucede, dónde, cuándo y con qué resultados. El propósito consistió en reagrupar los datos que se fueron obteniendo en el proceso anterior, para tener una explicación más precisa del fenómeno.

3. Por último, las categorías ligadas a subcategorías previamente definidas se relacionaron entre sí. A partir de esta relación se buscó mejorar la información recogida en las mismas y definir las categorías principales. Para facilitar este proceso según sugieren los autores Strauss y Corbin (2000), se realizaron diagramas y se tomaron notas relevantes, lo que permitió conocer cómo se estaba haciendo la relación de categorías y completar las que no se consideraron previamente como saturadas teóricamente. Este proceso descrito a partir de la recolección y la codificación se dio por concluido cuando se obtuvo toda la información en las categorías. 
Para realizar la codificación y análisis de la información antes descrita, se utilizó como apoyo el software de análisis cualitativo ATLAS.ti, el cual, según Chandra y Shang (2017) es una herramienta que facilita el proceso de codificación y análisis de la información, garantizando la transparencia y rigor de la investigación. Específicamente, este software permitió organizar una estructura compleja de categorías y subcategorías, garantizando el proceso de análisis, lo que se ajustó a las necesidades que se demandaban.

\subsection{Criterios para garantizar la validez}

Es necesario para las investigaciones cualitativas tener criterios propios de este tipo de proyectos, los que permitan demostrar que esta es rigurosa y creíble para la audiencia (Tracy, 2010; Anderson, 2017). Por lo tanto, a partir de los criterios que recogen Creswell y Miller (2000) para garantizar la validez de la investigación cualitativa se siguieron: la triangulación y la comprobación de miembros.

- Triangulación: Se utilizó para comparar la información recolectada entre los participantes (entre distintos trabajadores, y entre trabajadores y propietarios-gerentes). Garantizando de esta forma, descartar la información que sea contradictoria o no ofreciera certeza. Además, este método se manejó para comparar parte de la información recolectada en las entrevistas y contrastarla con las notas de la observación. De esta forma, se pudo verificar que lo que declaraban los participantes estaba en correspondencia con lo que se observó tanto en la capacitación formal como en la informal, específicamente en el diseño e implementación.

- Comprobación de miembros: Se mostraron a los propietarios-gerentes los resultados obtenidos: las estrategias y los elementos para el desarrollo de estas. Se discutieron con ellos estos hallazgos para ver su interpretación, lo que permitió un mejor entendimiento del fenómeno y una descripción más detallada.

\section{Resultados y discusión}

A partir de la metodología antes presentada, en este apartado se recogen los resultados obtenidos y su discusión con la literatura. Esta sección está compuesta por dos puntos fundamentales: los elementos para el desarrollo de estrategias de capacitación y las estrategias de capacitación identificadas.

\subsection{Elementos para el desarrollo de estrategias de capacitación}

Los elementos para el desarrollo de estrategias de formación en las pymes estudiadas, se agrupan en: agentes que intervienen en la capacitación, los facilitadores de la capacitación y el proceso que siguen. En el Esquema 1 se representan cada uno de los elementos y sus componentes, así como la relación existente. 


\section{Esquema 1}

Elementos para el desarrollo de estrategias de capacitación

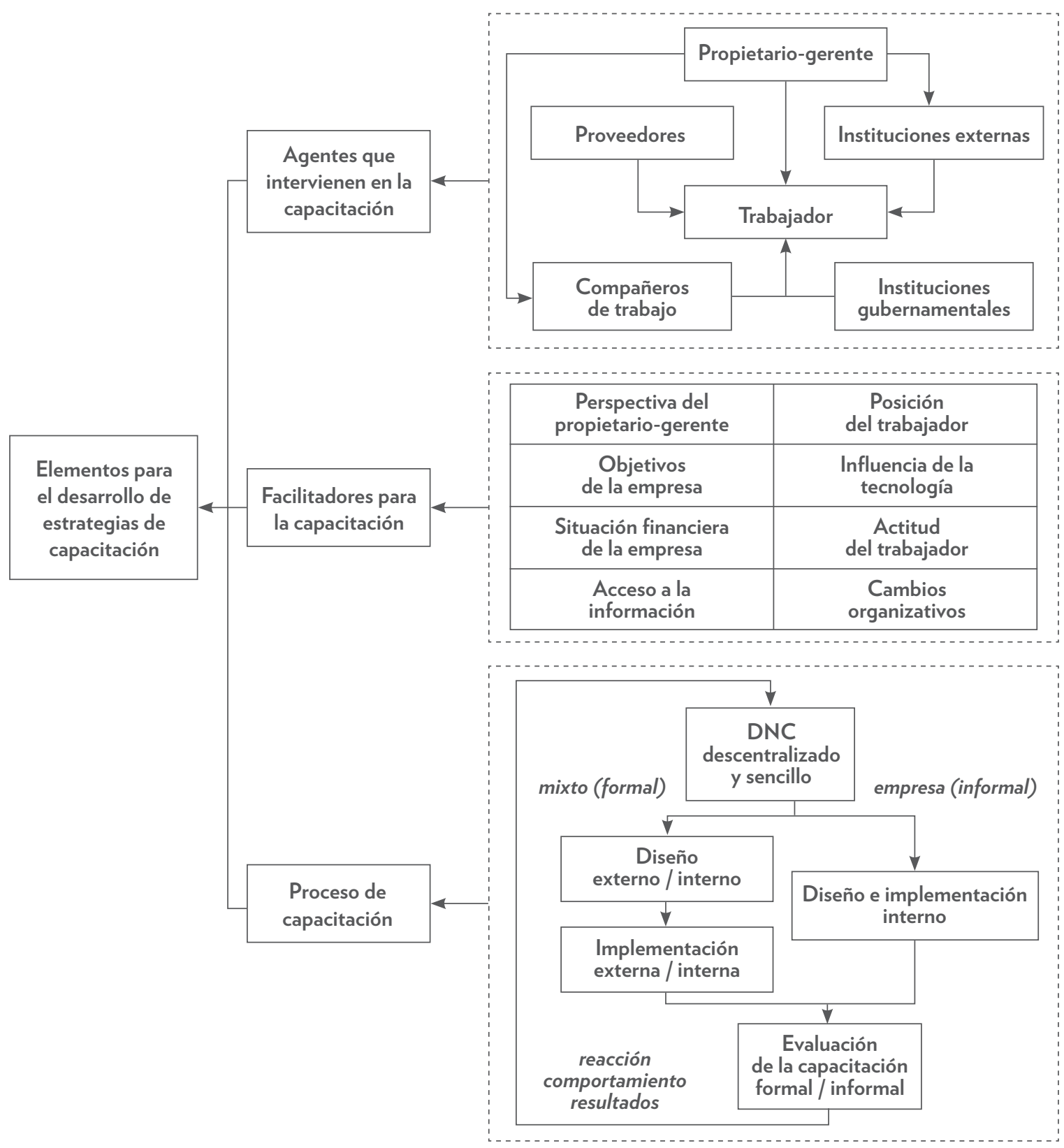

Fuente / Elaboración propia

\subsubsection{Agentes que intervienen en la capacitación}

Los agentes que intervienen en la capacitación están comprendidos por todas aquellas organizaciones y personas que influyen de forma directa en el proceso de capacitación de un trabajador. Estos son: el propietario-gerente, los proveedores (empresas grandes que suministran los productos que estas 
comercializan), instituciones externas (empresas que brindan servicios de capacitación), las instituciones gubernamentales, los compañeros de trabajo y el trabajador como elemento central. Los autores (Coetzer et al., 2019; Susomrith et al., 2019) encuentran al propietario-gerente como actor principal del aprendizaje informal. En este caso el propietario-gerente participa de la capacitación formal e informal y en estrecha relación con los otros agentes. Además, influye directamente en el trabajador a la hora de brindarle capacitación como instructor o indirectamente a través de la coordinación de capacitación con proveedores, instituciones externas y los compañeros de trabajo.

Si voy a capacitar a alguien, hay capacitación por Webinar, a través de las fábricas que representamos, con las que ya tenemos experiencia de capacitaciones anteriores. También los que ya están aquí y no tienen experiencia les enseño yo o les enseña el que ya sabe. (propietario-gerente, IM-E17)

Los compañeros de trabajo pueden intervenir por la acción del propietario-gerente, como se menciona anteriormente, o por su propia iniciativa. Al igual que en el estudio de Rabie et al. (2016), se destaca que estos son un elemento fundamental de apoyo en el aprendizaje, tanto en el momento de recibir la capacitación como después, cuando el trabajador realiza la transferencia de conocimiento al puesto de trabajo. "En equipo, como siempre hacemos, aquí siempre somos varios en las capacitaciones para poder ayudarnos... lo que alguno no sepa se lo puede consultar al compañero" (trabajador del taller, IM-E4).

Sobre los proveedores se observa que, coincidiendo con el estudio de De Fuentes y Dutrénit (2008), existen derramas de conocimientos por parte de estos a las pymes. Se evidencia a través de capacitaciones formales que tienen como propósito beneficiar a las pymes de conocimientos sobre los productos que les proveen, pues mientras estas sean capaces de vender más productos, las grandes empresas suministradoras también se verán beneficiadas. La capacitación en este caso, la cual es muy frecuente, se lleva a cabo de manera online, en el puesto de trabajo y por visitas de trabajadores a las grandes empresas. "Recibimos capacitación por parte de los proveedores, referente a los productos que manejamos para hacer el ensamble de los controles" (supervisor de talleres, IM-E1).

En el caso de las capacitaciones en instituciones gubernamentales, se realiza solo por trabajadores de áreas administrativas de la empresa. Los propietarios-gerentes no influyen en el trabajador para que la tome, aunque sí dan su aprobación.

\subsubsection{Facilitadores para la capacitación}

Se identifica un total de ocho elementos que detonan la ocurrencia de capacitación en estas pymes. A continuación, se describe cada uno.

- Perspectiva del propietario: En ambas empresas los propietarios-gerentes consideran la capacitación de suma importancia, dado que les permite obtener beneficios. Esto coincide con el estudio de Padachi y Bhiwajee (2016), el cual hace referencia a la visión de los dueños como elemento para el desarrollo de actividades de capacitación. Además, se observa que este participa no solo en el aprendizaje informal como, exponen Coetzer et al. (2019), sino también en el formal. 
- Objetivos de la empresa: Las metas que plantean estas pymes hacen que sea necesario dotar de conocimientos a los trabajadores. Ejemplo de esto es cómo la empresa IM envió un trabajador a capacitarse con un proveedor sobre determinado producto, para luego enviar a este a una sucursal en la que se comenzaría a comercializar el equipamiento nuevo. El origen de actividades de capacitación a partir de objetivos empresariales, está en relación con necesidades manifiestas según Mendoza (2005).

- Situación financiera de la empresa: Los propietarios-gerentes expresan que en ocasiones se ven limitados a realizar acciones de capacitación, por considerar elevado su costo. Pero también declaran que, si obtienen ganancias, realizan inversiones constantes en capacitación. "Para realizar capacitación miramos cómo va la empresa económicamente, si tenemos buenas ventas decidimos invertirle a la capacitación" (propietario-gerente, EJ-E1). Almeida y Aterido (2015) estiman que las pymes que declaran el factor económico como el más importante son las que tienen menos posibilidades de realizar capacitación laboral. Sin embargo, aunque ambas organizaciones tienen en cuenta el factor económico, realizan capacitaciones de forma frecuente y formal. Esto es contrario también a lo que plantean Padachi y Bhiwajee (2016), los que identifican una fuerte relación entre la tenencia de presupuesto para capacitación y la intención de realizar esta.

- Acceso a la información: Ambas organizaciones cuentan con información para tomar decisiones y conocer la oferta de capacitación formal disponible en el mercado. Ya sea a través de la que envían agentes como los proveedores o instituciones externas, o porque los propietarios-gerentes conocen dónde encontrarla. Almeida y Aterido (2015) identifican que el acceso a la información es un freno a las acciones de formación y añaden otros factores complementarios como la ubicación o el poco personal encargado de la gestión de las empresas. En el caso de ambas entidades económicas estudiadas, no se encuentran estos dos últimos factores que identifican estos autores.

- Puesto del trabajador: Se observa que se tienen prioridades a la hora de llevar a cabo la capacitación, pues los trabajadores que participan directamente de las actividades claves de las empresas (producción, ventas o servicios) declaran recibir capacitación constante, mientras que los trabajadores de apoyo (actividades administrativas) tienen muy poca o nula participación. El estudio de Padachi y Bhiwajee (2016) halla que el 25\% de los gerentes encuestados declaran brindar capacitación por las características técnicas del trabajo. Siendo esto mucho menor que el caso de las pymes estudiadas, donde la mayoría de la capacitación es técnica; lo que podría estar en relación por el sector al que estas se dedican.

- Influencia de la tecnología: Primero, se evidencia que los cambios tecnológicos como: nuevos diseños de equipos por parte de los proveedores o nuevas necesidades de clientes que suponen modificaciones tecnológicas en los productos, hacen que se desarrollen actividades de capacitación. Segundo, se halla que los trabajadores cuentan con acceso a la tecnología para el desarrollo de actividades de capacitación y a su vez para el apoyo de los conocimientos adquiridos una vez finalizada esta. Este último punto, Rabie et al. (2016) lo destacan como uno de los elementos fundamentales que refuerzan los conocimientos en las pymes. 
- Actitud del trabajador: Los empleados que demuestran una actitud positiva hacia el aprendizaje son más propensos a obtener formación, siempre que estos se desempeñen dentro de las actividades claves de la empresa. Uno de los gerentes de la empresa IM, considera que: "si el trabajador no está motivado por recibir capacitación no aprende y al final acaba en un puesto menor o abandonando la empresa" (propietario-gerente, IM-E17).

- Cambios organizacionales: Los cambios que se generan dentro de la organización influyen en la decisión de realizar capacitación, ejemplo de esto es: cuando hay trabajadores de nuevo ingreso, los cuales la gran mayoría no cuentan con los conocimientos necesarios, o cuando un trabajador abandona la organización e internamente debe ser remplazado. Esto coincide con lo planteado por Mendoza (2005), el cual lo identifica como una necesidad manifiesta de brindar capacitación.

\subsubsection{Proceso de capacitación}

De forma general predominan las cuatro etapas del proceso, siendo en las organizaciones los propietarios-gerentes los responsables de la coordinación de estas actividades, aunque los trabajadores de cada departamento intervienen. En el caso del diagnóstico se observa la presencia de las necesidades manifiestas y en menor medida de las encubiertas planteadas por Mendoza (2005). Siendo las formas más comunes de recolectar información la observación y las entrevistas, por los gerentes de cada área o por el propietario. También, los proveedores ofrecen capacitaciones sobre nuevos productos o cambios tecnológicos en equipos, lo que provoca que el diagnóstico ocurra de manera externa en este caso, aunque siempre contando con el visto bueno del dueño de la empresa. Sin embargo, al igual que en el estudio de Pauli (2019), se identifica que en estas empresas no se realizan programas de capacitación a largo plazo basado en el diagnóstico de sus necesidades. "Depende del área, pero en general nos la proponen o nosotros la proponemos cuando vemos que algo está fallando... es una necesidad que nace de cada departamento" (propietario-gerente, EJ-E1).

En el caso del diseño e implementación del programa de capacitación, este sucede de dos formas: de manera conjunta con alguna institución externa o proveedor, o solamente por la empresa de forma interna. Cuando ocurre esto último, ejemplo las capacitaciones aprendiz-maestro en el puesto de trabajo, se sigue un comportamiento que se define como informal, pues no se organiza y coordina de manera estructurada. Esta manera, de acuerdo con el estudio de Padachi y Bhiwajee (2016) es la forma más común de capacitación en las pymes. Por otra parte, cuando el diseño y la implementación se realiza con la intervención de la empresa y alguna institución externa o proveedor, se toma un carácter más formal. El estudio desarrollado por Rabie et al. (2016) señala que la mayoría de los trabajadores encuestados en las pymes recibieron capacitación de manera formal.

En las organizaciones estudiadas, existe una combinación de ambas formas de capacitación que se complementan para el desarrollo de conocimientos y habilidades en los trabajadores. Ejemplo de un carácter formal: "fue un curso en sala de juntas... venían de una a tres personas a impartirnos el curso apoyados con proyectores. También nos ponían videos y aparte nos explicaban lo que no entendíamos" (vendedora, IM-E11). De una forma más informal se encuentra: "se comentan entre los 
trabajadores, se comentan entre ellos como hacen las cosas, sobre todo en el área de producción, los más experimentados le dicen a los nuevos como hacer su trabajo" (propietario-gerente, EJ-E1).

En el caso de la evaluación, dependiendo de la forma en que se hizo el diseño e implementación, también va a ser el grado de rigurosidad de esta. Además, se puede ver que las empresas se enfocan a dos niveles en esta etapa, el comportamiento y los resultados en los indicadores económicos. Cuando la capacitación es impartida por agentes externos se halla que estos miden la reacción que tuvo el trabajador al curso, sin embargo, ni estos ni la empresa miden si el empleado obtuvo el aprendizaje. Aunque si existe una actitud favorable por parte de los trabajadores para ser evaluados. "Si estaría de acuerdo en que evaluaran la capacitación... así demostramos la capacidad que tenemos nosotros para solventar los problemas" (vendedor, IM- E12). Esto evidencia que están presente tres de los cuatro niveles de evaluación planteados por Kirkpatrick y Kirkpatrick (2006), pero contradice lo que sugieren estos autores, los cuales plantean que cada nivel es inviolable.

Además, se encuentra que no se mide el ROI cuando se realiza una inversión en capacitación. Sobre este tema, Sethi (2018) expresa que no tener un retorno favorable es una barrera para la capacitación. Sin embargo, en las pymes estudiadas no se observa evidencia de que esto sea un impedimento para realizar capacitación, aunque sí se considera importante que la formación influya en los indicadores de la impresa.

Hablando con ellos es como puedo saber que están contentos o que sirvió la capacitación que recibieron. También viendo si hacen bien su trabajo claro. Cuando hablas con ellos te dicen qué es lo que estaba mal con la capacitación que recibieron y qué es lo que les costó trabajo aprender o qué capacitación deberían tomar de nuevo. (propietario-gerente, IM-E18)

Alcanzar un proceso totalmente formal en las pymes según Pauli (2019) está dado por: los conocimientos de formación acumulados, la actitud del propietario-gerente y las limitaciones económicas. Sin embargo, aunque estas pymes cumplen en gran medida con estas características, existen procesos informales de capacitación, los cuales son un valioso complemento a la capacitación formal y al logro de los objetivos del negocio.

\section{2. $\mathcal{E}_{\text {strategias de capacitación }}$}

Todas las estrategias identificadas buscan dotar al trabajador de conocimientos y habilidades, con el menor gasto de recurso posible, siendo estos los dos elementos más importantes según declaran los participantes. Por lo tanto, las mismas se clasificaron de acuerdo a tres tipos: las que buscan el ahorro de recursos, las que se orientan de forma principal hacia conocimientos y habilidades y las que comprenden las dos anteriores en igual medida. En el Esquema 2, se representan las estrategias y subestrategias que la componen.

Las estrategias identificadas siguen una clasificación más amplia que la presentada por Bencsik y Sólyom (2011), pero tienen en cuenta la presencia de aprendizaje interno, aprendizaje externo y la combinación de ambos que sugieren estos autores. 
Esquema 2

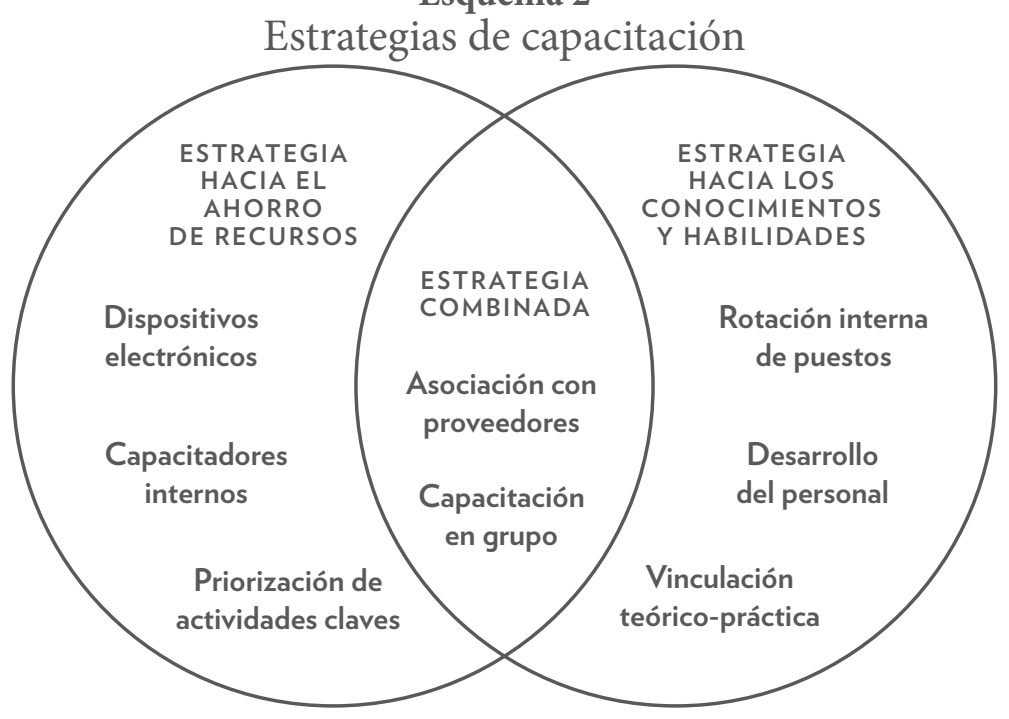

Fuente / Elaboración propia.

\subsection{1. $\mathcal{E}$ strategia hacia el ahorro de recursos}

Esta busca hacer frente al ahorro de recursos monetarios y de tiempo, elementos que se han identificado en la literatura como barreras a la capacitación ej. Bai et al. (2016); INEGI (2018) y Sethi (2018). Los tres tipos de subestrategias que conforman esta son: capacitadores internos, priorización de actividades claves y dispositivos electrónicos.

- Capacitadores internos: En las diferentes áreas de las empresas existen trabajadores que cuentan con una vasta experiencia dentro de la actividad que desempeñan. Estos fungen como capacitadores internos, los cuales imparten conocimientos a trabajadores de nuevo ingreso y a los trabajadores que son reubicados en nuevas áreas. Los criterios que se siguen para la selección de capacitadores internos son: que los trabajadores tengan experiencia, conocimientos y que se haya identificado una disposición de enseñar a otros. El propietario-gerente ejerce el papel de coordinador de estas actividades, pero también puede actuar como capacitador.

Esta subestrategia ocurre como parte del diseño e implementación internos del proceso de capacitación, por lo tanto, toma un carácter informal de aprendizaje. Sobre el tema, uno de los propietarios-gerentes declara: "los que están aquí y no tienen experiencia les enseño yo o les enseña el trabajador que ya sabe, yo me encargo en la parte técnica, en la otra parte se encarga el gerente administrativo" (propietario-gerente, IM-E17).

- Priorización de actividades claves: Cuando se decide llevar capacitación formal, estas empresas cuentan con un presupuesto limitado, el cual no está definido como parte de las inversiones. Por lo que se establece un orden de prioridades para aquellos trabajadores que reciben capacitación, teniéndose en cuenta la actividad que realiza el empleado. Aquellos miembros de la organización que desarrollan actividades denominadas claves, 
son los que reciben capacitación. Pues el resultado de acciones de capacitación, según los propietarios-gerentes, debe incidir directamente sobre los resultados económicos de la empresa. "Pues en la parte económica, que aumenten los ingresos, si el trabajador sabe puede hacer bien los productos o puede desarrollar habilidades para vender estos" (propietario-gerente, EJ-E1).

Por lo tanto, los trabajadores de las áreas de producción, servicio y ventas (actividades claves), están siendo capacitados frecuentemente.

En el último año fue constante, yo creo que fueron como unas 4 o 5 veces, vino un grupo de personas a la empresa, nos platicaron sobre temas como: resilientes, cómo tratar al cliente, temas psicológicos, etc. También, sobre un producto que manejamos de un proveedor vino una persona externa a capacitarnos. (vendedora, EJ-E3)

En cambio, los trabajadores de las áreas como contabilidad o almacenes (actividades de apoyo), declaran no recibir capacitación formal por parte de la empresa, aunque si de manera informal o gestionada por ellos mismos. "Yo recibí solamente dos capacitaciones pagadas por mí, sobre impuestos e impuestos federales el año pasado, a través de cursos presenciales" (contadora, IM-E8).

- Dispositivos electrónicos: Se identifica como principal ventaja el ahorro de tiempo por parte de los trabajadores, los cuales no deben desplazarse hacia los lugares donde se imparte la capacitación. Siendo, además, una solución al distanciamiento por la situación actual provocada por el Covid-19. "Las capacitaciones con dispositivos electrónicos ayudan mucho y disminuye mucho el tiempo, hay veces que no podemos ir lejos a tales lugares, estas plataformas ayudan, pues son muy completas" (jefe de compras, IM-E-3).

Aunque es una estrategia frecuente, se señala por parte de varios trabajadores que no es la forma en que prefieren aprender, debido al distanciamiento y las características propias de este método de aprendizaje, lo que puede ser una barrera para la absorción de conocimientos técnicos. "Con la pandemia hemos manejado capacitaciones a través de conferencias Web, ninguna presencial, no es igual para aprender, pero es lo que se puede por ahora" (trabajador del taller, IM-E4).

\subsection{2. $\mathcal{E}_{\text {strategia hacia conocimientos y habilidades }}$}

La estrategia que persigue principalmente dotar al trabajador de conocimientos y habilidades está compuesta por tres subestrategias: rotación interna de puestos, desarrollo del personal y vinculación teórico-práctica.

- Rotación interna de puestos: Busca distribuir el conocimiento clave en varios trabajadores y no centralizar este en un solo empleado o departamento. Esto sucede debido a que estas pymes, al no contar con un amplio personal, presentan problemas cuando suceden cambios organizativos que generan la ausencia de personal que domina cierto conocimiento, dígase: trabajadores que causan baja definitiva o temporal de la empresa; ya sea por vacaciones, enfermedad o finalización de contratos laborales. 
Esta subestrategia es adoptada, mayormente, para realizar capacitaciones internas y con un carácter informal, aunque en ocasiones también está presente en actividades formales de capacitación con proveedores o instituciones externas. Su principal objetivo es que no se detengan las actividades por la ausencia de conocimientos o habilidades de un trabajador.

Hemos intentado que todos los trabajadores se vayan involucrando en todas las funciones. En el supuesto caso de que alguien salga de vacaciones otros pueden asumir esas tareas y sacar el trabajo. Tratamos que el personal tenga los conocimientos básicos todos de todo, no que sea exclusivo solo de producción o que esta área solo sepa de embobinado. (propietario-gerente, EJ-E1)

- Desarrollo del personal: Este término se comprende en la literatura como preparación del trabajador para actividades futuras o un posible puesto (Noe, 2017). En estas pymes se sigue una idea similar, pues la mayoría de los trabajadores entrevistados declaran importante la capacitación para su permanencia en la empresa. Ven esta como una manera de apropiarse de conocimientos con una finalidad de crecimiento profesional y/o económico. Ejemplo de esto declara un trabajador de ventas de la empresa IM.

Estaría dispuesto aprender nuevas cosas para mi trabajo por motivaciones profesionales, para alcanzar otro puesto, otra oportunidad laboral... la pura capacitación no sería un factor determinante para permanecer en la empresa, tendría que venir acompañada con crecimiento dentro de la empresa y remuneración económica. (vendedor, IM-E2)

Por otra parte, estas dos empresas tienen bajos índices de fluctuación laboral. La mayoría de sus trabajadores tienen más de cinco años de permanencia. Los propietarios-gerentes identifican la necesidad de formación de sus trabajadores claves para un crecimiento dentro de la organización. Con este fin, destinan esfuerzos en retener y capacitar a los trabajadores, no solo para ocupar un puesto superior, pues esto se ve limitado en ocasiones por el tamaño de la empresa, sino para el desarrollo de más actividades en un mismo puesto de trabajo y por lo tanto una mayor remuneración. Estos hallazgos contrastan con la barrera para la implementación de capacitación que recoge Sethi (2018), quien encuentra como una de las causas que los propietarios-gerentes consideran que si se capacitan los empleados estos se marchan.

- Vinculación teórico-práctica: Las características propias de las actividades que desarrollan estas empresas, ya sea la venta de equipos o la fabricación de estos, hacen de la capacitación técnica la principal forma de aprendizaje. Independientemente de la formalidad de la capacitación, se entiende por los trabajadores y los propietarios-gerentes que la misma se debe realizar tanto de forma teórica como práctica. Pues mediante esta vinculación es como mejor se pueden desarrollar los conocimientos y habilidades necesarios en las actividades claves de la pyme. 
A veces hay buenos cursos tanto fuera como dentro de las empresas que fabrican materiales. A mí me tocó ir a Monterrey y ver como hacen las bombas, los equipos y como los prueban, es bueno tener esa experiencia. Cuando vienen los proveedores te dan un panorama explicado y puntual de ciertas cosas. (diseñador-vendedor, IM-E9)

En las prácticas de actividades informales, como es el caso de un trabajador de nuevo ingreso a la empresa, los propietarios-gerentes envían a estos a realizar actividades prácticas con los trabajadores más experimentados, luego de recibir explicaciones con apoyo en catálogos de fabricante o conocimientos del propio instructor. "Se comentan entre los trabajadores, se comentan entre ellos como hacen las cosas, sobre todo en el área de producción, los más experimentados le explican a los nuevos como hacer su trabajo" (propietario-gerente, EJ-E1).

Esta forma de capacitación mediante los conocimientos teóricos y prácticos coincide con lo planteado por los autores Rohayati y Wulandari (2017), quienes hallan que la principal forma en la que las pymes prefieren aprender es a través de demostraciones prácticas de cómo hacer determinada actividad.

\subsubsection{Estrategia combinada}

La misma está compuesta por dos subestrategias: la asociación con proveedores y la capacitación en grupo. Ambas estrechamente relacionadas, pero no presentes de forma conjunta en todas las ocasiones. La capacitación con proveedores se realiza mayormente en grupo, pero no toda la capacitación en grupo es realizada por los proveedores.

- Asociación con proveedores: Como se hace referencia sobre los agentes que intervienen en la capacitación, los proveedores de equipos de las pymes estudiadas en su mayoría grandes empresas, tienen un vínculo de proveeduría. Según Matus et al. (2018) esto ocurre porque las pymes se insertan en las cadenas de suministros hacia adelante y hacia atrás, como suministradores o comercializadores. Entre estas organizaciones estudiadas y las grandes empresas proveedoras existe una relación comercial formal. Estas últimas suministran equipos y brindan conocimientos como consultores o mediante capacitaciones formales, lo que tiene como finalidad que las pymes tengan éxito al comercializar los productos. Estas capacitaciones pueden ser para la fabricación de accesorios o sobre las características de los equipos. Mediante esta asociación las pymes garantizan acciones formales de capacitación constante y sin costo. Estas capacitaciones permiten la oportunidad de cumplimiento de los objetivos empresariales, con base en una necesidad de capacitación manifiesta.

Tenemos proveedores de equipos que nos brindan capacitación, principalmente en el área de servicios y ventas, ellos vienen a dar capacitación de los productos que manejan. Puede ser que ellos la ofrecen o nosotros se la solicitamos y ellos vienen. (propietario-gerente, EJ-E1)

En la empresa IM esta es la principal forma en la que se realiza capacitación formal, pues están estrechamente relacionados con los fabricantes de equipos de bombeo de líquidos. "En el 2019, como 
llevamos instrumentación, nos dieron capacitaciones de varias marcas... sobre todo, la parte de bombas. También nos dieron de otras marcas que no comercializamos mucho...pero casi todo relacionado con bombas industriales" (vendedora, IM-E11).

- Capacitación en grupo: Es frecuente que las actividades de capacitación se realicen de forma conjunta, donde participen varios miembros de la organización, de manera que se socialice el conocimiento entre los trabajadores. En las capacitaciones formales asisten todos los trabajadores que pertenecen a actividades claves que están relacionadas entre sí. Los propietarios-gerentes buscan que todos tengan dominio de los conocimientos por dos razones. Primero, que todos los trabajadores que asistan a esta capacitación puedan realizar y dominen una actividad en común. Segundo, que los trabajadores que no adquirieron determinado conocimiento puedan preguntar a otros que funjan de consultores internos.

La capacitación la realizamos en grupo, individual no sería efectiva. A veces cuando no sabes le preguntas a los compañeros y si ellos no la tomaron también no te van a poder ayudar si algo no lo entendiste u olvidaste como hacerlo (trabajador de taller, IM-E5).

Mediante estas capacitaciones en grupo, se trata que asista la mayor cantidad de trabajadores que se relacionan con el tema impartido, pues así no será necesario repetirla. Esto ocurre independientemente si esta se imparte por un proveedor o porque se contrate a una institución externa. "Prefiero en grupo, personal no, todos aquí para sacarnos de dudas a todos...todos asistimos o casi todos cuando vienen a impartirla aquí" (trabajador de servicios, EJ-E5).

\section{Conclusión}

Las pymes estudiadas adoptan varias estrategias con la finalidad de llevar a cabo capacitaciones. Estas se identifican que se orientan a los conocimientos y las habilidades, y/o el ahorro de recursos. $\mathrm{Al}$ desarrollar las estrategias mencionadas, estas organizaciones encuentran una forma de superar las barreras para realizar capacitación que afrontan las pymes mexicanas; obteniendo así beneficios tanto para la empresa como para sus trabajadores. Como complemento de las estrategias, se identifica además que para el desarrollo de las mismas están presentes tres elementos: los agentes que intervienen en la capacitación, los habilitadores para la ocurrencia y el proceso que siguen. Se destaca como relevante que estas entidades económicas tienen al propietario-gerente como un agente fundamental, pero no es el único involucrado en impulsar las acciones de capacitación, los compañeros de trabajo las instituciones externas y los proveedores juegan un roll determinante en materia de capacitación en estas pymes. Además, se entiende que el proceso de capacitación informal es un complemento de las actividades de aprendizaje formal, pues permite a las empresas: distribuir el conocimiento internamente y el ahorro de recursos. Por último, las estrategias de capacitación identificadas y los elementos que componen el desarrollo de estas, pueden ser una guía que permita a otras pymes una solución al desarrollo de actividades de formación. 


\section{Referencias}

Almeida, R. K., y Aterido, R. (2015). Investing in formal on-the-job training: are SMEs lagging much behind? IZA Journal of Labor and Development, 4(8), 1-23. https://doi.org/10.1186/ s40175-015-0029-3

Anderson, V. (2017). Criteria for evaluating qualitative research. Human Resource Development Quarterly, 28(2), 1-9. https://doi.org/10.1002/hrdq.21282

Aragón-Sánchez, A., Barba-Aragón, I., y Sanz-Valle, R. (2003). Effects of training on business results. International Journal of Human Resource Management, 14(6), 956-980. https:/doi. org/10.1080/0958519032000106164

Ayyagari, M., Demirguc-Kunt, A., y Maksimovic, V. (2014). Who creates jobs in developing countries? Small Business Economics, 43(1), 75-99. https://doi.org/10.1007/s11187-014-9549-5

Bai, Y., Yuan, J., y Pan, J. (2016). Why SMEs in emerging economies are reluctant to provide employee training: Evidence from China. International Small Business Journal, 35(6), 751-766. https:// doi.org/10.1177/0266242616682360

Barrett, R. (2015). Small firm training: Just meeting the day-to-day needs of the business. Employee Relations, 37(5), 547-567. https://doi.org/10.1108/ER-05-2014-0048

Bencsik, A., y Sólyom, A. (2011). Strategies of education and training practice of small and medium sized enterprises. International Journal of Educational and Pedagogical Sciences, 5(3), 263-269. https://doi.org/10.5281/zenodo.1331481

Birks, D. F., Fernandez, W., Levina, N., y Nasirin, S. (2013). Grounded theory method in information systems research: Its nature, diversity and opportunities. European Journal of Information Systems, 22(1), 1-8. https://doi.org/10.1057/ejis.2012.48

Blaxter, L., Hughes, C., y Tight., M. (2002). Como se hace una investigación (2nd ed.). Gedisa.

Blume, B. D., Ford, J. K., Baldwin, T. T., y Huang, J. L. (2010). Transfer of training: A meta-analytic review. Journal of Management, 36(4), 1065-1105. https://doi.org/10.1177/0149206309352880

Chandra, Y., y Shang, L. (2017). An RQDA-based constructivist methodology for qualitative research. Qualitative Market Research, 20(1), 90-112. https://doi.org/10.1108/QMR-02-2016-0014

Ciriaci, D. (2016). Intangible resources: the relevance of training for European firms' innovative performance. Economia Politica, 34(1), 31-54. https://doi.org/10.1007/s40888-016-0049-8

Congreso General de los Estados Unidos Mexicanos. (2012, Noviembre 11). Decreto por el que se reforman, adicionan y derogan diversas disposiciones de la Ley Federal del Trabajo. Diario Oficial de la Federación. http://www.dof.gob.mx/nota_detalle.php?codigo=5280815\&fecha=30/11/2012

Coetzer, A., Wallo, A., y Kock, H. (2019). The owner-manager's role as a facilitator of informal learning in small businesses. Human Resource Development International, 22(5), 420-452. https://doi.or $\mathrm{g} / 10.1080 / 13678868.2019 .1585695$

Corbin, J., y Strauss, A. (1990). Grounded theory research: procedures, canons and evaluative criteria. Zeitscrift Für Soziologie, 19(6), 418-427. https://doi.org/10.1007/BF00988593

Corbin, J., y Strauss, A. (2002). Bases de la investigación cualitativa. Técnicas y procedimientos para desarrollar la teoría fundamentada. Universidad de Antioquia. 
Creswell, J. W. (2014). Research Design (4th ed.). Sage.

Creswell, J. W., y Miller, D. L. (2000). Determining validity in qualitative inquiry. Theory into Practice, 39(3), 124-130. https://doi.org/10.1207/s15430421tip3903_2

Crowe, M., y Sheppard, L. (2012). Mind mapping research methods. Quality and Quantity, 46(5), 1493-1504. https://doi.org/10.1007/s11135-011-9463-8

De Fuentes, C. y Dutrénit, G. (2008). Diferencias de los mecanismos de derramas de conocimiento en dos localidades mexicanas. Economía y Sociedad, 14(22), 47-69.

Dessler, G., y Varela, R. (2011). Administración de recursos humanos (5th ed.). Pearson Education.

Devins, D., y Johnson, S. (2003). Training and development activities in SMEs: Some findings from an evaluation of the ESF objective 4 programme in Britain. International Small Business Journal, 21(2), 213-228. https://doi.org/10.1177/0266242603021002005

Eisenhardt, K. M. (1989). Building theories from case study research. Academy of Management Review, 14(4), 532-550. https://doi.org/10.2307/258557

Gainey, T. W., y Klass, B. S. (2003). The outsourcing of training and development: Factors impacting client satisfaction. Journal of Management, 29(2), 207-229. https://doi.org/10.1016/S01492063(02)00215-5

Hodges, T. (2002). Linking Learning and Performance. Butterworth- Heinemann.

Independent Evaluation Group. (2019). World Bank Group support for small and medium enterprises: A synthesis of evaluative findings. World Bank. https://openknowledge.worldbank.org/handle/10986/32536

Instituto Nacional de Estadística y Geografía. (2018). Encuesta nacional sobre productividad y competitividad de las micro, pequeñas y medianas empresas. Instituto Nacional de Estadística y Geografía. https://www.inegi.org.mx/programas/enaproce/2018/

Instituto Nacional de Estadística y Geografía. (2020). Estadísticas a propósito del día de las micro, pequeñas y medianas empresas. Instituto Nacional de Estadística y Geografía. https://www.inegi. org.mx/contenidos/saladeprensa/aproposito/2020/MYPIMES20.pdf.

Jabbour, C. J. C. (2013). Environmental training in organizations: From a literature review to a framework for future research. Resources, Conservation and Recycling, 74(1), 144-155. https://doi. org/10.1016/j.resconrec.2012.12.017

Jain, S. (2014). Methods of training programmes evaluation: A review. The Journal of Commerce, 6(2), 19-30.

Jehanzeb, K., y Bashir, N. A. (2013). Training and development program and its benefits to employee: A conceptual study. European Journal of Business and Management, 5(2), 243-252.

Kotey, B., y Folker, C. (2007). Employee training in SMEs: Effect of size and firm type - family and nonfamily. Journal of Small Business Management, 45(2), 214-238. https://doi.org/10.1111/ j.1540-627X.2007.00210.x

Matus, M., Carrillo, J., y Gomis, R. (2018). Empresas multinacionales, derramas de conocimiento y spin-off en México ¿El país de origen hace la diferencia? Perfiles Latinoamericanos, 26(52), 1-21. https://doi.org/10.18504/pl2652-007-2018

Mendoza, A. (2005). Manual para determinar las necesidades de capacitación y desarrollo (5th ed.). Trillas. 
Mondy, W. R. (2010). Administración de recursos humanos (11th ed.). Pearson.

Noe, R. A. (2017). Employee training and development (7th ed.). McGraw-Hill.

Nolan, C. T., y Garavan, T. N. (2016a). Human resource development in SMEs: A systematic review of the literature. International Journal of Management Reviews, 18(1), 85-107. https://doi. org/10.1111/ijmr.12062

Nolan, C. T., y Garavan, T. N. (2016b). Problematizing HRD in SMEs: A "critical" exploration of context, informality, and empirical realities. Human Resource Development Quarterly, 27(3), 1-36. https://doi.org/10.1002/hrdq.21261

Oficina Internacional del Trabajo. (2015). Pequeñas y medianas empresas y creación de empleo decente y productivo. Oficina Internacional del Trabajo. https://www.ilo.org/ilc/ILCSessions/previous-sessions/104/reports/reports-to-the-conference/WCMS_358292/lang--es/index.htm

Organización Mundial del Comercio. (2016). Informe sobre el comercio mundial 2016: Igualdad de condiciones para el comercio de las pymes. Secretaría de la Organización Mundial del Comercio. https://www.wto.org/spanish/res_s/booksp_s/world_trade_report16_s.pdf

Ostrowski, B., Kolomitro, K., y Lam, T. C. M. (2014). Training methods: A review and analysis. Human Resource Development Review, 13(1), 11-35. https://doi.org/10.1177/1534484313497947

Padachi, K., y Bhiwajee, S. L. (2016). Barriers to employee training in small and medium sized enterprises - insights and evidences from Mauritius. European Journal of Training and Development, 40(4), 1-18. https://doi.org/10.1108/EJTD-02-2014-0018

Pauli, U. (2019). Training professionalization and SME performance. Human Resource Development International, 23(2), 168-187. https://doi.org/10.1080/13678868.2019.1696079

Rabie, C., Cant, M. C., y Wiid, J. A. (2016). Training and development in SMEs: South Africa's key to survival and success? The Journal of Applied Business Research, 32(4), 1009-1024. https://doi. org/10.19030/jabr.v32i4.9717

Rohayati, Y., y Wulandari, S. (2017). Training needs analysis for MSMEs: how to improve training effectiveness. IOP Conf. Series: Materials Science and Engineering, 277(1), 1-9. https://doi.org/ doi:10.1088/1757-899X/277/1/012030

Secretaría de Economía. (2009, Junio 30). Acuerdo por el que se establece la estratificación de las micro, pequeñas y medianas empresas. Diario Oficial de la Federación. http://dof.gob.mx/nota_detalle.php? codigo $=5096849 \&$ fecha $=30 / 06 / 2009$

Sethi, A. (2018). Obstacles faced by small medium enterprises in imparting training: an empirical study on textile industry. Pacific Business Review International, 10(10), 103-109.

Short, H. J., y Gray, D. E. (2018). HRD in SMEs: A research agenda whose time has come. Human Resource Development Quarterly, 29(1), 1-7. https://doi.org/10.1002/hrdq.21305

Siliceo, A. (2004). Capacitación y desarrollo de personal (4th ed.). Limusa.

Smit, Y., y Watkins, J. A. (2012). A literature review of small and medium enterprises (SME) risk management practices in South Africa. African Journal of Business Management, 6(21), 6324-6330. https://doi.org/10.5897/AJBM11.2709

Stake, R. E. (1999). Investigación con estudio de casos (2nd ed.). Morata.

Susomrith, P., Coetzer, A., y Ampofo, E. (2019). Training and development in small professional services firms. European Journal of Training and Development, 43(5/6), 517-535. https://doi. org/10.1108/EJTD-11-2018-0113 
Tao, Y. H., Yeh, C. R., y Sun, S. I. (2006). Improving training needs assessment processes via the Internet: System design and qualitative study. Internet Research, 16(4), 427-449. https://doi. org/10.1108/10662240610690043

Tracy, S. J. (2010). Qualitative quality: Eight “big-tent” criteria for excellent qualitative research. Qualitative Inquiry, 16(10), 837-851. https://doi.org/10.1177/1077800410383121

Vásquez-Torres, M. del C. (2018). Factorial analysis of the elements that compose the training in the small and medium enterprises of the industrial sector. Management, 22(2), 42-59. https://doi. org/10.2478/manment-2018-0022

Werther, W., y Davis, K. (2008). Administración de recursos humanos. El capital humano de la empresa (6th ed.). McGraw Hill. 